\title{
Evaluation of Age Group Swimmers: A in Field Proposal
}

\author{
Pedro Morouço*, José Sacadura, Nuno Amaro and Rui Matos
}

Research Center for Human Movement Sciences, Polytechnic Institute of Leiria, Leiria, Portugal

\begin{abstract}
Swimming evaluation it's the most appropriate procedure to analyze the development of a swimmer since age group swimming. The training control provides tools to prescribe intensities and volumes of training, but also assesses the improvement of each swimmer throughout that prescription. In the season 2007/2008 Leiria' Swimming Association had 419 swimmers competing by 13 Swimming Clubs. A selection process through 3 different stages was taken and evaluations were processed according to the swimmer development.
\end{abstract}

Keywords: Development, performance, critical velocity, tethered swimming, anthropometry.

\section{INTRODUCTION}

Several benefits can be obtained from a program that aims to evaluate the different factors that influence the swimmer performance $[1,2]$. The knowledge of the different components of multidimensional development and their role in sportive performance are fundamental factors in training young swimmers towards the elite performance [3]. Thus, we may assume that performance does not result from an isolated component, i.e., it is not enough to ensure strength or flexibility despising technique or vice versa [4].

A swimmer must live a transformation period from a multidisciplinary and general approach towards a specific enhancement of swimming technique. The purpose of this study is to present a proposal of evaluation, for age group swimmers taking into consideration the different factors that influence performance in swimming.

\section{METHODS}

Throughout the years we have been developing a system to select swimmers according to best performances, differing in techniques and distances, associated to age and years of practice. From this analysis, every year swimmers are selected for: 1st stage (boys under 12 years and girls under 11 years of age), the period of evaluation occur in 1 weekend and 1 swimming event; 2nd stage (boys under 14 years and girls under 13 years of age), the evaluation period occur in 1 weekend plus 2 swimming events; and 3rd stage (boys under 16 years and girls with 14 years of age), evaluation period occurs in 2 weekends plus participating in 2 swimming competitions.

\section{1st Stage}

In this first stage we focus our interest in swimming technique evaluation (4 strokes, turns and starts) using qualitative analysis sheets and video analysis. Complemen-

*Address correspondence to this author at the Research Center for Human Movement Sciences, Polytechnic Institute of Leiria, Leiria, Portugal;

Tel: +351 915754483; Fax: +351 244829499;

E-mail: pmorouco@esel.ipleiria.pt tarily, anthropometric evaluation consists in measurement of: weight; height; span; length of the upper limb; length of the lower limb; length and breadth of the hand; length and breadth of the foot; bi acromial breadth; bi iliac crest breadth and sum of 6 skinfolds. The functional assessment consists in vertical jump height; hands strength and sit and reach test; and psychological approach is conducted with TEOSQ and PMQ.

\section{2nd Stage}

In 2nd stage it is conducted a similar analysis to 1st stage plus a maximum effort of front crawl swimming for $200 \mathrm{~m}$ and 400m assessing Critical Velocity (CV).

\section{3rd Stage}

At this pre-junior level, swimmers tend to became specialists in a certain distance or stroke, therefore, the qualitative analysis is made for the 2 best strokes, turns and starts. Additionally, it is used $30 \mathrm{~s}$ tethered swimming test to assess an estimator of anaerobic capacity of the swimmer.

\section{Qualitative Analysis}

In order to evaluating swimming technique an apparatus was built for the purpose. This apparatus is capable of providing images of the swimmer from sagital and horizontal planes at the same time enhancing a more reliable analysis of swimming technique, turns and starts. In order to avoid different interpretations 5 sheets were produced; one to each stroke plus one for starts and turns. These sheets are adaptable to each stage being more specific as the swimmer gets older. At each evaluation period two swimming coaches evaluate each swimmer and then data is crossed in order to assess a uniform evaluation.

\section{Quantitative Analysis}

\section{Critical Velocity}

There are different protocols validated to predict the performance in long events, i.e., mainly using the aerobic energetic system. Nonetheless, the validation and usage of these protocols in young athletes is scarce. Among the most re- 
ferred in swimming literature we find the individual anaerobic threshold and the maximum consumption of oxygen. In order to avoid the complicated procedures and highly costs of this type of evaluation, Wakayoshi et al. [5] advanced with the term of CV. Hill [6] stated that CV is a valid indicator of aerobic performance not being influenced by the age of the swimmer. However, this statement is not supported using this methodology with age group swimmers, especially to what concerns the distance to swim in each effort.

\section{Tethered swimming}

Despite this methodology does not evaluate the drag that the swimmer must overcame, it is able to analyze the swimmer's capacity of using energy to produce strength. In addition, one of the main restrictions to higher performances is fatigue, being one of the main aspects to consider when training and competing. Nonetheless, the mechanics of fatigue appearance (and the way to delay it) remains not completely understood. Briefly, after an $800 \mathrm{~m}$ low intensity warm-up, each swimmer performs a $30 \mathrm{~s}$ maximum intensity front crawl test tethered to a load-cell that is able to export the data in ASCII format to a computer (Globus, Italy). From this methodology it is possible to assess individual force to time curves, calculating average and maximum force production, fatigue index, fatigue slope, coefficient of variation and differences in bilateral force production.

\section{RESULTS AND DISCUSSION}

In Table 1 are displayed illustrative mean $( \pm S D)$ values for anthropometric and functional variables assessed, according to stage and gender.

In Fig. (1) it is possible to observe an example of a representative curve of the tethered swimming test, showing the decrease of force production along the 30 s effort.

Table 1. Some Results of Anthropometric and Functional Data According to Gender and Stage of Evaluation. *Significant for $p<0.05$ Between Gender

\begin{tabular}{|c|c|c|c|}
\hline & Boys & Girls & Total \\
\hline $1^{\text {st }}$ stage & Mean \pm SD & Mean \pm SD & Mean \pm SD \\
\hline Body Mass (Kg) & $38,4 \pm 5,40$ & $38,6 \pm 6,02$ & $38,5 \pm 5,64$ \\
\hline Height (cm) & $147,0 \pm 7,62$ & $144,8 \pm 6,05$ & $145,9 \pm 6,85$ \\
\hline Body Mass Index & $17,6 \pm 1,28$ & $18,4 \pm 2,23$ & $18,0 \pm 1,87$ \\
\hline bi acromial breadth $(\mathrm{cm}) *$ & $36,7 \pm 3,08$ & $38,7 \pm 2,21$ & $37,7 \pm 2,82$ \\
\hline bi iliac crest breadth $(\mathrm{cm})$ & $29,3 \pm 1,74$ & $30,0 \pm 1,69$ & $29,7 \pm 1,72$ \\
\hline bi acromial/bi iliac crest & $1,3 \pm 0,09$ & $1,3 \pm 0,08$ & $1,3 \pm 0,09$ \\
\hline Foot length & $22,6 \pm 1,24$ & $22,3 \pm 0,89$ & $22,5 \pm 1,07$ \\
\hline Foot breadth & $8,3 \pm 0,58$ & $8,1 \pm 0,59$ & $8,2 \pm 0,58$ \\
\hline SUM 6 Skinfolds $(\mathrm{mm}) *$ & $55,4 \pm 23,31$ & $79,9 \pm 26,58$ & $67,7 \pm 27,56$ \\
\hline Left hand strength (Kgf) * & $21,0 \pm 3,58$ & $17,3 \pm 3,13$ & $19,1 \pm 3,81$ \\
\hline Right hand strength (Kgf) * & $22,6 \pm 3,71$ & $18,5 \pm 2,92$ & $20,5 \pm 3,90$ \\
\hline Vertical jump height $(\mathrm{cm})$ & $23,6 \pm 4,23$ & $22,1 \pm 3,64$ & $22,8 \pm 3,95$ \\
\hline Sit and reach $(\mathrm{cm}) *$ & $-3,0 \pm 7,95$ & $3,3 \pm 4,09$ & $0,3 \pm 6,94$ \\
\hline \multicolumn{4}{|l|}{$2^{\text {st }}$ stage } \\
\hline Maximum Force (s) * & $283.6 \pm 45.02$ & $196.8 \pm 29.38$ & $248.9 \pm 58.33$ \\
\hline Fatigue Index (\%) & $41.4 \pm 8.29$ & $38.6 \pm 5.57$ & $40.4 \pm 7.33$ \\
\hline
\end{tabular}




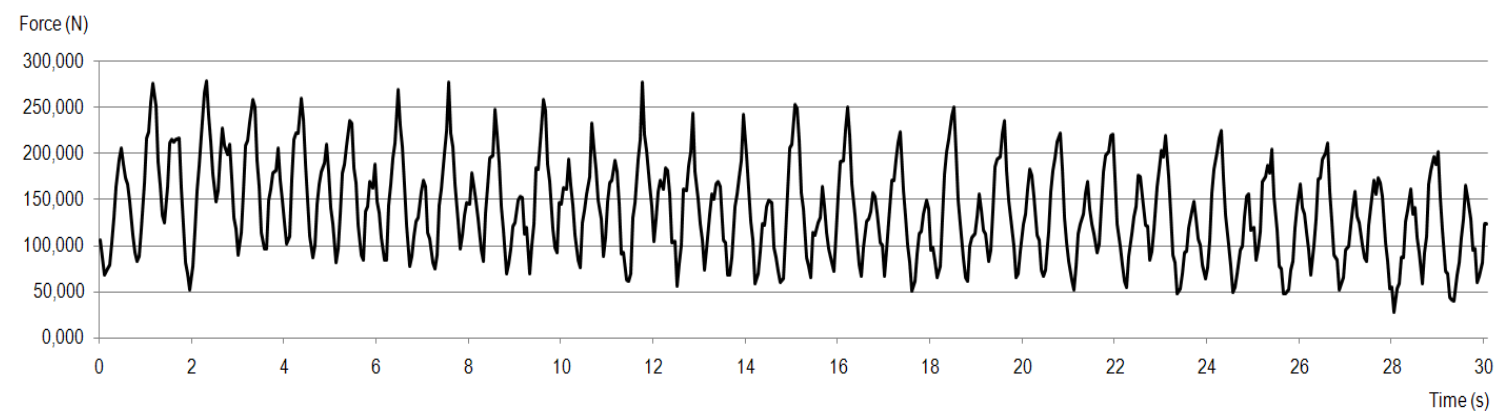

Fig. (1). Individual F(t) curve obtained through a front crawl tethered test.

Cumulative data collected throughout the years allows us to define range of values of successful and unsuccessful swimmers at elite level. Taking these data in consideration it is given the swimmer a punctuation from 1 to 5 : 1 if he presents a value smaller than a; 2 presenting a value between a and $b ; 3$ between $b$ and $c ; 4$ between $c$ and $d$; and 5 if it is higher than d. For instance the results obtained from Critical Velocity provide us the ideal velocities for development of aerobic resistance. In mean terms, the group presented in Table 1 should train trials of $400 \mathrm{~m}$ at 5:47, 83 for boys and $6: 03,64$ for girls. However, this test is individual and, thus, the calculation of time according to distance is identified for each swimmer separately. We have been doing efforts trying to clarify the values assessed through tethered swimming $[7,8]$ because we believe that it can be a reliable procedure to evaluate a swimmers anaerobic capacity and to predict performance in sprint and middle distance events.

Swimming testing and evaluation have been usually conducted in different fields of Sport Sciences [9]. However, nowadays, it seems difficult to define a barrier among those areas as they join efforts in order to achieve the ultimate scope: to help coaches in improving athlete's performance. The proposal presented seems to be a valid methodology to evaluate that age group swimmers as it focus in main components that may influence performance in swimming.

\section{REFERENCES}

[1] Mujika I, Chatard JC, Busso T, Geyssant A, Barale F, Lacoste L. Effects of training on performance in competitive swimming. Can J Appl Physiol 1995; 20(4): 395-406.

[2] Stager J, Tanner D, Eds. Swimming - handbook of sports medicine and science - 2nd ed. Oxford: Blackwell Publishing 2004.

[3] Rama L, Santos J, Gomes P, Alves F. Determinant factors related to performance in young swimmers. Port J Sport Sci 2006; 6(2): 246-49.

[4] Reilly T, Morris T, Whyte G. The specificity of training prescription and physiological assessment: a review. J Sports Sci 2009; 27: 575-89.

[5] Wakayoshi K, Yoshida T, Udo M, et al. A simple method for determining critical speed as swimming fatigue threshold in competitive swimming. Int J Sports Med 1992; 13: 367-71.

[6] Hill D. Aerobic and anaerobic contributions in middle distance running events. Motriz 2001; 7: 63-7.

[7] Morouço P, Soares S, Vilas-Boas JP, Fernandes R. Relationship between tethered swimming forces and front crawl and butterfly performances. In: Cabri J, Alves F, Araújo D, Barreiros J, Diniz D, Veloso A, Eds. European college of sport science, $13^{\text {th }}$ Annual Congress, Abstract Book; Estoril, Portugal 2008; p. 214.

[8] Morouço P, Soares S, Vilas-Boas JP, Fernandes R. Correlation of tethered swimming with swimming performance. Arch Med Dep 2008; 128: 512.

[9] Fernandes R. Physiological, cineanthropometrical and psychological characterization of pre-junior swimmer. MSc. Dissertion., Dept. of Swimming, OPorto University 1999.

(C) Morouço et al.; Licensee Bentham Open.

This is an open access article licensed under the terms of the Creative Commons Attribution Non-Commercial License. (http://creativecommons.org/licenses/by-nc/3.0/) which permits unrestricted, non-commercial use, distribution and reproduction in any medium, provided the work is properly cited. 